\title{
Aplicación de la fotogrametría con drones al control deformacional de estructuras y terreno.
}

\author{
Application of drone photogrammetry to the control of deformations of terrain \\ and structures
}

$\underline{\text { Rubén Sancho Gómez-Zurdo }}^{(*)(*)(* * *)}$, David Galán Martín ${ }^{(* *)(* * * *)}$, Beatriz González-Rodrigo ${ }^{(* * * *)}$, Miguel Marchamalo Sacristán ${ }^{(*)}$, Rubén Martínez Marín ${ }^{(*)}$

\section{RESUMEN}

En este trabajo se estudia la viabilidad del empleo de drones para el control de deformaciones del terreno y las estructuras, analizando para cada caso, la resolución, precisión y validación con otras técnicas. Se presenta con detalle la técnica fotogramétrica Structure From Motion, empleada para elaborar ortofotografías y modelos $3 \mathrm{D}$ precisos sin necesidad de conocer previamente las posiciones y ángulos de incidencia. El uso de puntos de control precisos, así como drones con sistema integrado RTK, son factores relevantes para la obtención de resultados con alta precisión. Se presenta el caso de estudio de la monitorización de una gran estructura, en este caso una presa arco-gravedad. Los resultados obtenidos presentan una precisión en deformaciones de $\pm 2 \mathrm{~mm}$ para la estructura. Esto confirma que la fotogrametría dron es aplicable al control deformacional de presas de hormigón, abriendo las posibilidades a la monitorización de otras grandes estructuras e infraestructuras.

Palabras clave: UAV; RPAs; fotogrametría; SFM; control de estructuras; monitoreo de presas; monitoreo de laderas

\section{ABSTRACT}

This work studies the viability of the use of drones for deformational control of terrain and structures, analyzing for each case the resolution, precision and validation with other techniques. The Structure From Motion photogrammetric technique is presented in detail, allowing the production of accurate orthophotographs and $3 D$ models without knowing previously positions and angles of incidence. The use of precise control points (Ground Control Points) as well as drones with integrated Real Time Kinetic "RTK" system, are relevant factors for obtaining high precision results. The case study of the monitoring of a large structure is presented, in this case an arch-gravity dam. The initial results show a precision in deformations of $\pm 2 \mathrm{~mm}$. This paper confirms that drone photogrammetry is applicable to the deformational control of concrete dams, opening the possibilities to the monitoring of other large structures and infrastructures.

Keywords: UAV; RPAs; photogrammetry; SFM; structural control; dam monitoring; slope monitoring

(*) Lab. Topografía y Geomática. ETSI Caminos, Canales y Puertos Universidad Politécnica de Madrid, Madrid, España ${ }^{* *}$ ALTIUS GEOTECNIA S.L. / IDEA INGENIERIA S.L. Zaragoza, España

$\left({ }^{* * *}\right)$ Comité Español de Grandes Presas (SPANCOLD). Madrid, España

$\left({ }^{* * * *}\right)$ Universidad Alfonso X el Sabio. Villanueva de la Cañada. Madrid, España

(*****) U.D Resistencia de Materiales. ETSI Civil. Universidad Politécnica de Madrid, Madrid, España

Persona de contacto/Corresponding author: rsancho@ingenieriaidea.com (R. Sancho)

ORCID: https://orcid.org/oooo-0oo2-1801-9418 (R. Sancho Gómez-Zurdo); https://orcid.org/oooo-0001-6589-6643 (D. Galán Martín); http://orcid.org/oooo-0oo3-1038-7459 (B. González-Rodrigo); http://orcid.org/oooo-ooo1-9237-4146 (M. Marchamalo); http://orcid. org/oooo-0oo2-2433-9354 (R. Martínez)

Cómo citar este artículo/Citation: Sancho Gómez-Zurdo, Rubén; Galán Martín, David; González-Rodrigo, Beatriz; Marchamalo Sacristán, Miguel; Martínez Marín, Rubén (2021). Aplicación de la fotogrametría con drones al control deformacional de estructuras y terreno. Informes de la Construcción, 73(561): e379. https://doi.org/10.3989/ic.77867

Copyright: (C) 2021 CSIC. Este es un artículo de acceso abierto distribuido bajo los términos de la licencia de uso y distribución Creative Commons Reconocimiento 4.0 Internacional (CC BY 4.0). 


\section{INTRODUCCIÓN}

Las nuevas tecnologías aplicadas al control deformacional, como son el GPS diferencial, laser escáner, radar terrestre etc., se basan en la toma de datos desde un punto fijo. Este hecho, limita su aplicación en zonas de difícil acceso y limitada visibilidad. Asimismo, no todos los puntos observados se encuentran en iguales condiciones de distancia, ángulo de incidencia, luminosidad etc. pudiendo afectar estas condiciones a la precisión del resultado final.

Los nuevos sistemas de captura de imágenes e información desde cualquier ubicación, han favorecido el avance y mejora de sistemas clásicos de medición como es la fotogrametría. En este sentido, la aparición de equipos capaces de llevar diferentes sensores y ser pilotados por control remoto "RPAs o drones" ha hecho posible que la inspección de fachadas, paramentos y en general grandes estructuras, se puedan realizar con un alto grado de detalle.

\subsection{Los drones}

La palabra "dron" (en plural "drones") procede del inglés "drone" y tiene la traducción literal al castellano como "abeja macho" derivado del zumbido que este produce al volar. La Agencia Estatal de Seguridad Aérea (AESA) describe como dron o RPA (Remotely Piloted Aircraft) las aeronaves pilotadas por control remoto, también enmarcadas en el grupo de vehículos aéreos no tripulados "VANT" (1), conocidos como UAV (Unnamed Aerial Vehicle) en inglés.

Atendiendo a su arquitectura, los drones se pueden clasificar principalmente en dos categorías: ala fija y multirrotores. El Plan Estratégico de Drones del Ministerio de Fomento de 2018 establece una clasificación más detallada (1).

\subsection{Tendencias generales}

El desarrollo de los drones y por tanto su utilidad y diversidad de aplicación, ha crecido exponencialmente en los últimos años (2), lo que ha provocado la necesidad de elaborar una normativa específica, así como la obligatoriedad de que los pilotos de drones obtengan licencias específicas y que las empresas que trabajen con drones se acrediten cómo "operadoras aéreas". Todo ello mediante escuelas acreditadas ATO (Approved Training Organization) y la propia AESA (Agencia Estatal de Seguridad Aérea).

La evolución de esta nueva tecnología y, por tanto, el uso de los drones en gran diversidad de ámbitos, se refleja de forma directa en el número creciente de artículos técnicos publicados en los últimos años. El número de publicaciones sobre UAV y drones se ha triplicado en los últimos cuatro años llegando a más de 1500 publicaciones anuales en Web of Science en 2017 (3).

El sector de las infraestructuras supone más del 35\% de las inversiones del mercado de drones (4), destacando la correspondiente al mantenimiento de las mismas tanto en edificación como en grandes infraestructuras, donde forman parte las presas.

Un segundo indicador del desarrollo de esta nueva tecnología, es la previsión de inversión que cada país hace y tiene previsto hacer en este sector. Goldman Sachs, líder a escala global en banca de inversión, presentó en 2018 una previsión económica de inversiones por países desde 2017 hasta 2021 (5), en la que Estados Unidos llega a los 17,5 millones de dólares, China a los 4,5 y Reino Unido 3,5 millones de dólares invertidos en este sector.

Los nuevos sistemas inteligentes aplicados a la construcción, como el Building Information Modelling "BIM", se consideran parte de la "revolución digital" dentro de este sector (4), y están directamente relacionados con los drones, incluyendo:

1. Modelos 3D casi instantáneos.

2. Monitoreo del proceso constructivo en tiempo real.

3. Imágenes y ortofotografías de alta precisión.

Los nuevos desarrollos tanto en los propios drones, como en los sensores, sistema de transmisión de datos entre la estación y en el dron, y las mejoras de posicionamiento en tiempo real (RTK / PPK), permiten conseguir precisiones inferiores al centímetro, tal y como se presenta en este artículo.

\subsection{La fotogrametría con drones con la técnica "Structure From Motion".}

La fotogrametría tradicional requiere que las imágenes adquiridas tengan posiciones y ángulos de incidencia conocidos. Por el contrario, con la técnica Structure From Motion (SFM) se pueden emplear fotografías tomadas desde distintos ángulos y distancias, incluso sin ser conocida su posición (6)(7). De esta forma, el desarrollo de los vehículos aéreos no tripulados (drones), la mejora y reducción de peso de diferentes sensores (especialmente las cámaras tipo RGB) junto con la adecuada estabilización de los mismos, con sistemas inerciales de 3 ejes, ha hecho que la fotogrametría clásica haya sido sustituida en gran medida por la técnica SFM (8).

Así, con la posibilidad de adquirir imágenes desde cualquier posición y orientación, la fotogrametría con drones ha evolucionado en nuevas aplicaciones. En este sentido, el uso de la fotogrametría con drones en el ámbito de la ingeniería, es ya una realidad con aplicaciones en el visible y el térmico (9).

En el caso de la seguridad de presas, la combinación de imágenes y vídeos junto con la obtención de modelos georreferenciados y escalados en los que se puedan realizar mediciones (Figura 1), constituye una característica importante de esta técnica. Esto, junto con la competitividad económica que ofrece, lo convierte en una tecnología con grandes posibilidades.

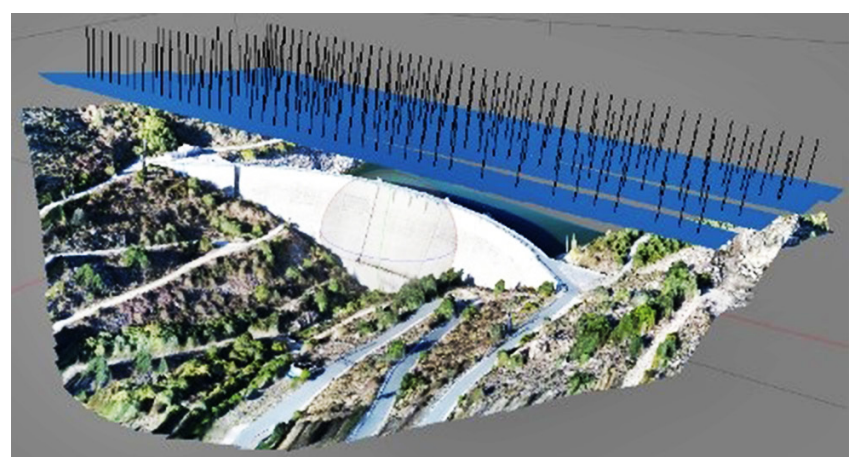

Figura 1: Modelo 3D y puntos de captura de imágenes en vuelo con dron de presa arco-gravedad. 
La mejora de la velocidad de cálculo de los nuevos ordenadores y el desarrollo computacional de la fotogrametría (10), ha hecho que, junto con la evolución de los drones, se obtengan resultados de mayor calidad y mejor precisión. En esa línea, el desarrollo de softwares basados en SFM (Tabla 1) permite la obtención de nubes de puntos y modelos $3 \mathrm{D}$ con gran rapidez.

El fundamento teórico de la técnica SFM (11) se basa en la superposición de imágenes para obtener el modelo $3 \mathrm{D}$, es decir, a partir de, al menos dos imágenes que tengan pixeles comunes (homólogos), se obtiene la posición y orientación de la cámara, así como las coordenadas del punto correspondiente al pixel en cuestión.

Con los nuevos algoritmos, la obtención de dichas nubes de puntos a partir del proceso SFM, se lleva a cabo en base a las correspondencias detectadas entre las imágenes. Estas correspondencias o emparejados se realizan mediante la transformación característica de escala invariante (SIFT) (12), que permite dicho emparejado incluso con grandes variaciones de escala, posición e incluso iluminación. Estos parámetros influyen en el grado de precisión deseado.

Como se ha dicho, una de las ventajas que caracteriza a la técnica SFM es que no es necesario que las imágenes a partir de las cuales se realiza el procesado, estén referenciadas, escaladas etc. por lo que, de ser así, el modelo $3 \mathrm{D}$ resultante estará referenciado en coordenadas relativas.

Esto no implica que no se pueda obtener un modelo georreferenciado en coordenadas absolutas. Es aquí, donde se incorpora el concepto de Puntos de Apoyo o Ground Control Points (GCPs). Dotando a estos puntos de apoyo de unas coordenadas conocidas (GCPs), se obtiene el modelo a partir de la técnica SFM en coordenadas absolutas.

A continuación, se procede a desarrollar la técnica SFM de forma sucinta y "paso a paso" según las fases propuestas por (6).

\section{Paso 1}

Para llevar a cabo la reconstrucción de la escena, se comienza por la búsqueda de los puntos de interés (keypoints), que son comparados entre sí para obtener correspondencias (matching) entre las imágenes. La aproximación más utilizada para llevar a cabo este paso es la técnica SIFT (transformación de la escala invariante) (12) que permite ganar calidad

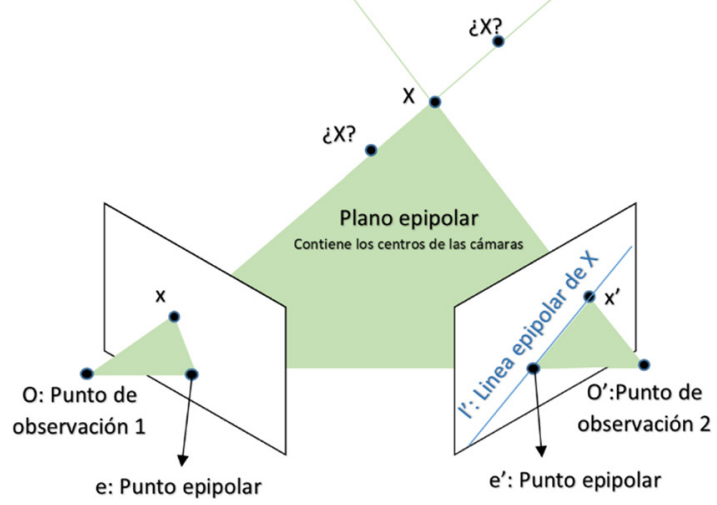

Figura 2: Geometría epipolar. Elaboración propia basada en (10). en los resultados. El objetivo es obtener el movimiento entre las cámaras, es decir, medir las distancias entre ellas en el plano de imagen de la cámara.

Desde los puntos de observación O y O' (centros de cámara), se tiene que la proyección de O' se ve en la imagen 1, concretamente en el punto e (epipolo). De forma análoga se obtiene e' y si esto no sucede es que una cámara no observa a la otra. Extrapolando esta deducción a la proyección de todos los puntos comprendidos en OX, se obtiene en la imagen 2 la línea l', línea epipolar. El plano formado por los centros de las cámaras $\mathrm{O}$ y O' y el punto X obtenido por triangulación, es el plano epipolar (Figura 2).

La obtención de estas correspondencias entre imágenes para conseguir la orientación y posición de las cámaras (en coordenadas relativas), requiere, además, de la matriz fundamental (F) y la matriz esencial (E). La matriz fundamental, es una matriz de $3 \times 3$ de segundo rango que realiza el mapeo de un punto de la primera imagen a uno de la línea epipolar de la segunda (Figura 2). De esta forma, dado el plano epipolar P, los puntos x y x' (proyecciones del punto X en ambas imágenes en coordenadas homogéneas) y al estar x' en la línea epipolar l' se tiene (Hartley \& Zisserman, 2000) (Figura 2):

$$
\mathrm{l}^{\prime}=\left[\mathrm{e}^{\prime}\right] \cdot \mathrm{H}_{\mathrm{P}} \cdot \mathrm{x}=\mathrm{F} \cdot \mathrm{x}
$$

La matriz fundamental cumple la condición que para cada par de puntos x, x':

$$
\mathrm{x}^{T} \cdot \mathrm{F} \cdot \mathrm{x}=0
$$

Lo cual es cierto ya que si para cada (x, x'), x' se encuentra sobre línea epipolar l'=F·x correspondiente al punto $\mathrm{x}$, se tiene:

$$
\mathrm{x}^{T} \cdot \mathrm{l}^{\prime}=0=\mathrm{x}^{T} \cdot \mathrm{F} \cdot \mathrm{x}
$$

De aquí, se obtiene que para n correspondencias entre imágenes se verifica (10):

$$
\left(\begin{array}{ccc}
\left(X \cdot X^{\prime}\right)_{1} & \ldots & 1 \\
\cdots & \ldots & \ldots \\
\left(X \cdot X^{\prime}\right)_{n} & \ldots & 1
\end{array}\right) \cdot\left(\begin{array}{c}
f_{11} \\
\ldots \\
f_{33}
\end{array}\right)=0
$$

En la matriz fundamental, las coordenadas de los puntos de la imagen vienen definidos en píxeles. Para que queden expresados en coordenadas normalizadas, hay que introducir el concepto de matriz esencial (E), a partir de la cual se puede obtener las matrices de proyección de las cámaras. Definiendo $\mathrm{v}$ como la inversa del punto $\mathrm{x}$ a través de una matriz $\mathrm{K}$ conocida, la matriz esencial se define por la ecuación:

$$
\mathrm{v}^{T} \cdot \mathrm{E} \cdot \mathrm{v}=0
$$

Para las correspondencias x y x' expresado en términos de imágenes con coordenadas normalizadas, se puede expresar:

$$
\begin{aligned}
\mathrm{v}^{T} & =\mathrm{v}^{\prime} \cdot \mathrm{K}^{T} \\
\mathrm{v} & =\mathrm{K}^{-1} \cdot \mathrm{v}
\end{aligned}
$$

Que se pueden sustituir en la ecuación anterior dando lugar a:

$$
\mathrm{v}^{T *} \mathrm{~K}^{\prime T} \cdot \mathrm{E} \cdot \mathrm{K}^{-1} \cdot \mathrm{v}=0
$$


Finalmente se obtiene:

$$
\mathrm{E}=\mathrm{K}^{T} \cdot \mathrm{F} \cdot \mathrm{K}
$$

A partir de la matriz esencial, se obtienen las matrices de proyección de las cámaras mediante su descomposición en dos matrices ( $\mathrm{W}$ de traslación y $\mathrm{Z}$ de rotación). De la factorización de la matriz esencial se calcula el vector de traslación de la matriz de proyección (P) suponiendo los autovalores de la matriz esencial E unitarios.

Con la información anterior se obtiene el punto $\mathrm{X}$, que es aquel cuya posición minimiza el error o distancia entre sus proyecciones en las dos imágenes y los puntos de correspondencia (Figura 3).

Como resultado de todos estos procesos, se calculan las ubicaciones coincidentes (puntos homólogos) de varios puntos en dos o más fotografías, para los que normalmente hay una única solución matemática que indica dónde se tomaron dichas fotografías. Así, se obtienen las posiciones de cámara (x, $\mathrm{y}, \mathrm{z}),\left(\mathrm{x}^{\prime}, \mathrm{y}^{\prime}, \mathrm{z}^{\prime}\right)$, orientaciones j, j', longitudes focales o, o', y posiciones relativas $\mathrm{m}, \mathrm{n}$ (Figura 4 ).

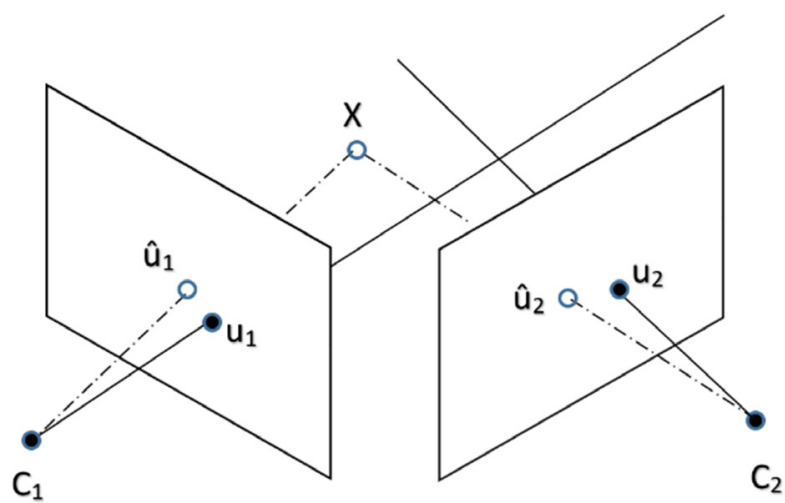

Figura 3: Geometría epipolar. Triangulación y error de proyección. Elaboración propia basada en (10).

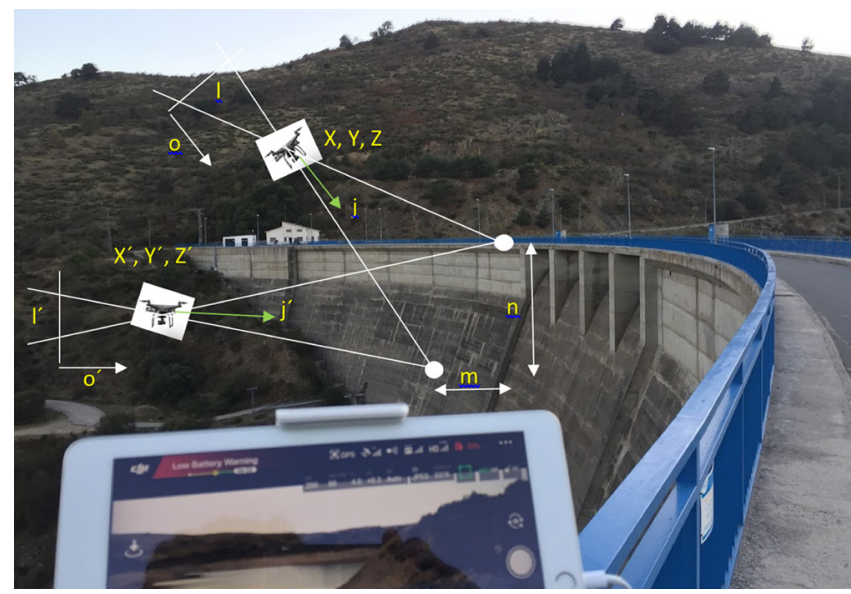

Figura 4: Posiciones de cámara, orientación, longitudes focales y posiciones relativas.

Paso 2

Como se ha visto anteriormente, la obtención de esta "única” solución matemática lleva consigo una serie de procesos y transformaciones, que junto con el "ruido" de las imágenes utilizadas en el proceso, da lugar a posibles errores de repro- yección (Figura 5). Este error se debe minimizar en la mayor parte de lo posible, lo cual se lleva a cabo mediante un ajuste denominado "Bundle adjustment" (o "Ajuste por Haces").

El "Ajuste por Haces" es una optimización con la finalidad de refinar las posiciones de la cámara y las coordenadas $3 \mathrm{D}$ de los puntos. Matemáticamente se define como el método no lineal para minimizar los errores de la proyección que calcula la distancia sobre la imagen entre el punto proyectado " $\mathrm{x}$ " $\mathrm{y}$ medido "X":

$[10]$

$$
e=\sum_{i=1}^{n} \sum_{j=1}^{m} d\left(P_{i} X_{j}, x_{i j}\right)^{2}
$$

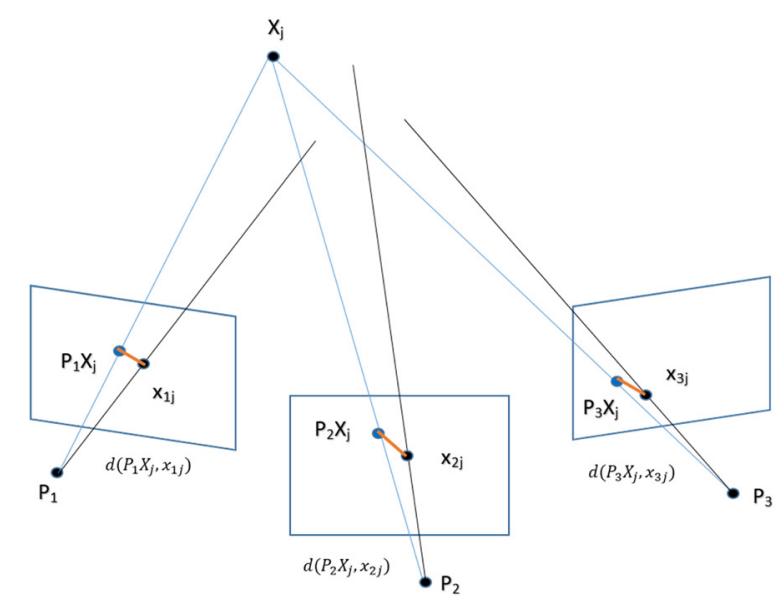

Figura 5: Error de proyección d: Matriz de Proyección P, punto proyectado X y su proyección $\mathrm{x}$.

En cada ajuste, la matriz jacobiana se puede separar en los cálculos, hecho que en combinación con el procesado en paralelo mediante los nuevos softwares (Tabla 1), hace que se puedan resolver trabajos mediante SFM de gran cantidad de imágenes.

\section{Paso 3}

A continuación, se determina la nube de puntos densa y una superficie $3 \mathrm{D}$ utilizando los parámetros de cámara conocidos y utilizando los puntos obtenidos.

De forma genérica, todos los píxeles de las imágenes se utilizan para que la nube de puntos densa (Figura 6) sea lo más similar posible en resolución, a las fotografías, aunque algunos softwares hacen distinción y solo utilizan píxeles con una cierta redundancia. Este paso recibe el nombre de "Multi View Stereo Matching” (MVS).

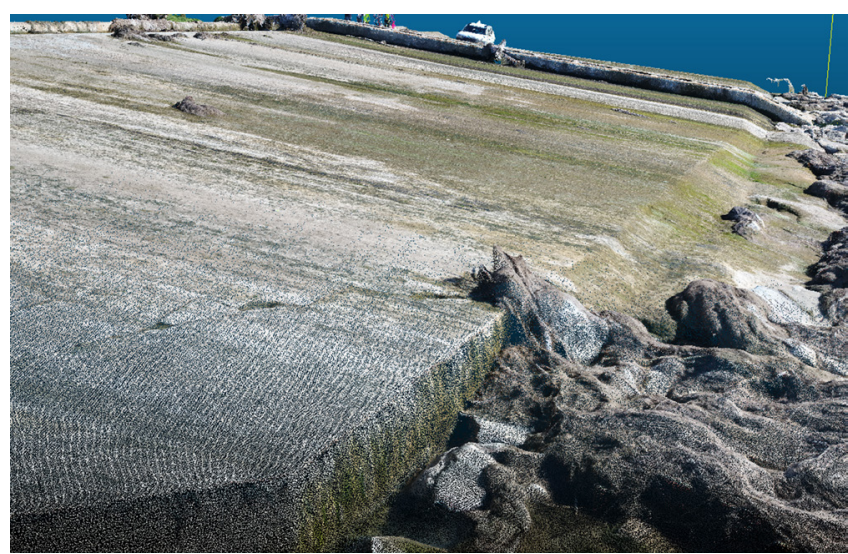

Figura 6: Nube de puntos densa en modelo realizado en Azud con $955 \mathrm{pts} / \mathrm{m}^{2}$. 
Paso 4

La Georectificación significa convertir la nube de puntos desde un sistema de coordenadas arbitrario en un sistema de coordenadas geográficas. Esto se puede lograr de dos maneras:

1. Directamente, con el conocimiento de las posiciones de la cámara y las longitudes focales (posible en drones con sistema RTK)

2. Indirectamente, incorporando puntos de control de tierra (GCP) con coordenadas conocidas. Típicamente estos serían adquiridos usando GPS o Estación Total.

\section{Paso 5}

Aun no siendo un paso intrínseco de la técnica SFM, si es habitual su obtención como culminación del mismo. Por ello se incluye como paso final la obtención del Modelo Digital de Elevaciones, Ortofoto y Modelo texturizado.

\subsection{Posicionamiento en tiempo real: RTK / PPK.}

El RTK (Real Time Kinetic) o geoposicionamiento automático en tiempo real de cada imagen tomada por el dron, hace que la simple comparativa de imágenes en dos periodos de tiempo, tengan la misma perspectiva, con un error en posición de 2-4 cm. De esta forma, incluso sin necesidad de llegar a establecer complejos modelos 3D mediante fotogrametría se puede tener una rápida comparativa para analizar la evolución de la zona de la infraestructura estudiada.

\subsection{La auscultación con drones}

El seguimiento, vigilancia y auscultación de una gran estructura, tiene como punto de partida la inspección visual de la misma. Por definición, la auscultación con drones parte de esa premisa, debido a que es a partir de las imágenes tomadas en el vuelo, con las que se inicia el trabajo y procesado de las mismas. Mediante programas comerciales como los indicados en la Tablas 1 y 2, se obtiene la nube de puntos y modelo $3 \mathrm{D}$ en color verdadero.

La comparativa de dos modelos, en diferentes instantes de tiempo, permite obtener las diferencias entre ambos, pero si además están georreferenciados en el mismo sistema de coordenadas, se obtienen desplazamientos y/o deformaciones.

De esta manera, la mejora de los propios drones unida al desarrollo de los softwares de procesado, implica que la inspección, vigilancia, análisis y control de movimientos de las grandes estructuras con drones, sea una realidad en casos como:

1. Estructuras: Inspección, mapeo y control de deformaciones en edificación, presas y puentes.

2. Taludes y laderas:

a. Inspección y control de movimientos en deslizamientos, vuelcos etc. tanto en suelos como en macizos rocosos.

b. Análisis de estabilidad mediante la obtención de: dirección de buzamiento, buzamiento de grietas y fracturas para su análisis estereográfico y consecuente análisis de estabilidad.

3. Situaciones de emergencia: terremotos, inundaciones, incendios etc.

\subsection{Objetivos}

El objetivo fundamental de esta investigación consiste en desarrollar una metodología innovadora para el control de movimientos, en estructuras y taludes, mediante técnicas fotogramétricas basadas en adquisiciones de imágenes con dron. La validación de esta metodología se realizará mediante comparación con técnicas clásicas de auscultación. Además, se analizan los condicionantes y limitaciones de la misma y se aplica a un estudio de caso en una presa arco-gravedad.

\section{ESTADO ACTUAL DEL CONTROL DE MOVI- MIENTOS Y DEFORMACIONES CON DRONES}

Se ha realizado una búsqueda bibliográfica sistemática, en los portales, editoriales y medios de publicación académicos y profesionales, para poder presentar el estado de esta técnica aplicada al control de movimientos, particularmente de laderas y presas.

Los motores de búsqueda en los que se realizó la búsqueda fueron: Web of Science, Google Scholar, Researchgate repositorios

Tabla 1. Programas de cálculo con técnica Structure From Motion.

\begin{tabular}{|c|c|c|c|}
\hline Compañía & Origen & Software & Licencia \\
\hline Agisoft & Rusia & Photoscan & Comercial \\
\hline Pix4d & Suiza & Pix4d & Comercial \\
\hline Bentley & EEUU & Context Capture & Libre \\
\hline Microsoft Live Labs & EEUU & Photosynth & Libre \\
\hline Changchang Wu (Google) & EEUU & VisualSFM & \\
\hline
\end{tabular}

Tabla 2. Programas de tratamiento de nubes de puntos y modelos 3D.

\begin{tabular}{|c|c|c|c|}
\hline Compañía & Pais & Software & licencia \\
\hline Phd Daniel Girardeau-Montaut & France & Cloud Compare & Comercial \\
\hline Leica & EEUU & 3D Reshaper & Comercial \\
\hline Bentley & EEUU & Pointools & Comercial \\
\hline InnovMetric & Canadá & Polyworks & Comercial \\
\hline Autodesk & EEUU & Civil3D & \\
\hline
\end{tabular}


de editoriales como ScienceDirect y Springer Link y revistas especializadas como Sensors y Remote Sensing. Las palabras clave utilizadas en la búsqueda fueron: "drone-RPAS-UAVUAS-dam" "drone-RPA-UAV-UAS-reservoir", "photogrammetry-SFM-dam”, "drone RPA-UAV-UAS-survey", "drone RPAS-UAV-UAS-monitoring” "drone-RPA-UAV-UAS-photogrammetry", "drone-RPA-UAV-UAS-landslide", "photogrammetry-SFM-landslide". Con este procedimiento se obtuvieron un total de 34 artículos en los que se utilizan drones en tareas de inspección, mapeo, monitoreo y control de deformaciones y en concreto presas y laderas.

\subsection{Aplicación a presas, laderas y macizos rocosos}

Los artículos se han resumido y ordenado atendiendo al país donde se aplica la técnica (Figura 7), año de publicación (Figura 8) y su tipología (Figura 9).

Al tratarse de una nueva tecnología, las publicaciones de los artículos relacionados son recientes. De esta forma se puede concluir que, la extensión del uso de drones en aspectos relacionados con la seguridad de presas y laderas, ha tenido lugar en los últimos cinco años (Figura 8).

\subsection{Desarrollo por países}

En primer lugar, se encuentran con seis artículos cada uno, Italia $(13,14,15,16,17,18)$ y Austria $(19,20,21,22,23,24)$, aunque bien es cierto que en el caso de Italia varios de ellos son referentes a la Presa Ridracoli $(13,16,17)$. A continuación se sitúan China $(25,26,27,28,29)$ y EEUU $(30,31,32,33,34)$, siendo los norteamericanos los únicos que cuentan con un documento oficial "Monitoring Levees" (30) en el que se hace referencia explícita al uso de drones en la seguridad de presas.

España, a pesar de tener más de 1200 grandes presas e innumerables balsas, presenta tres artículos siendo además dos de ellos correspondientes a laderas $(35,36)$. El más reciente incluye un análisis interesante de dos configuraciones SFM (37).

Como puede observarse, y a pesar de ser una técnica relativamente reciente, ya se ha utilizado en varios países, probablemente por su bajo coste y la expansión general de las tecnologías basadas en drones.

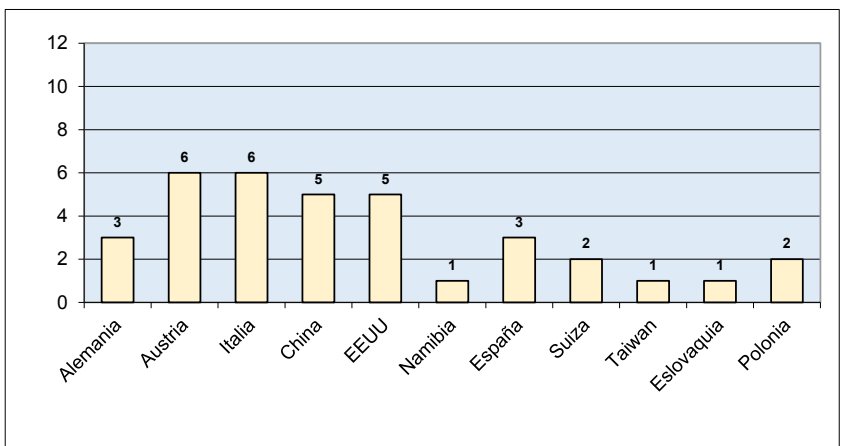

Figura 7: Clasificación de artículos científicos sobre drones y auscultación de infraestructuras hidráulicas por país de aplicación.

\subsection{Evolución temporal y por campos de aplicación}

La evolución de las publicaciones es un buen análisis indicativo de la tendencia de una técnica. En este sentido, y dentro del sector de la inspección y auscultación de estruc- turas, y en concreto de las presas, se puede observar que su mayor desarrollo se ha producido en los últimos cinco años. El desarrollo e inversión tecnológica actual y futura de los drones, sensores y software de procesado, junto con la necesidad de mejora en materia de seguridad de las estructuras, hace prever que se dispare a corto plazo la aplicación de esta técnica, tal y como se puede apreciar en la Figura 8.

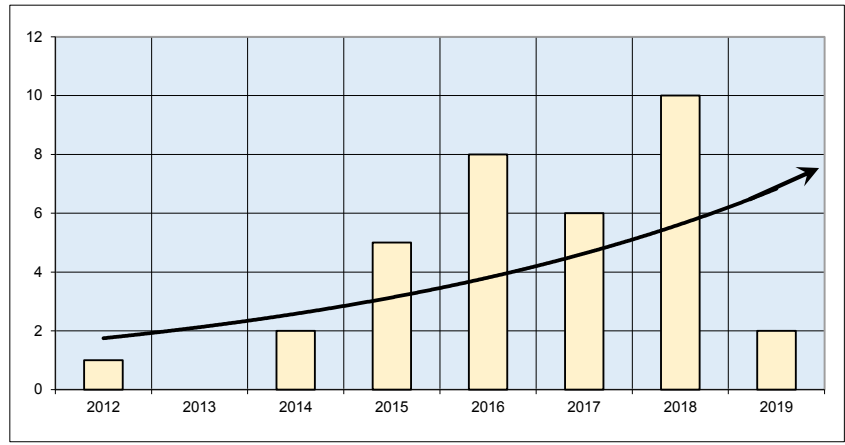

Figura 8: Clasificación de artículos científicos sobre drones y auscultación de infraestructuras hidráulicas por año de publicación. Nota. 2019 está incompleto

En el caso de aplicación a presas y laderas, las publicaciones se han clasificado en tres grandes grupos según se puede observar en la Figura 9. Estos son: el relacionado con las estructuras tipo "Presa", el aplicado a la gestión y calidad de embalses "Gestión del Agua" y el que hace referencia a laderas "Laderas".

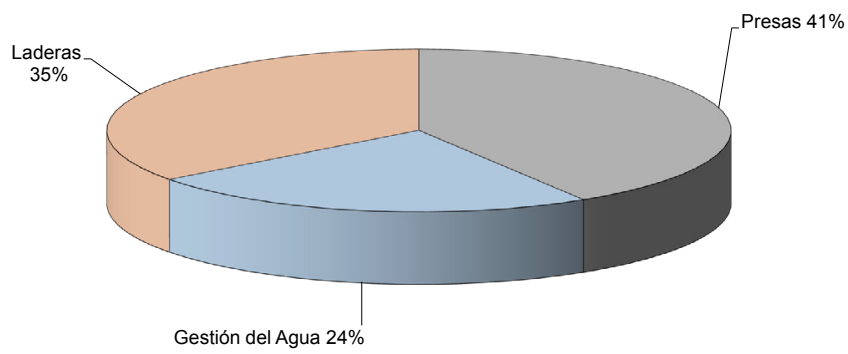

Figura 9: Clasificación de artículos científicos sobre drones y auscultación de infraestructuras hidráulicas por tipología de aplicación.

\section{ESTUDIO DE CASO. APLICACIÓN AL CONTROL DE GRANDES INFRAESTRUCTURAS: PRESAS DE HORMIGÓN}

\subsection{Trabajo de campo}

En el caso de estudio, los vuelos se realizaron sobre una presa Arco-Gravedad, utilizándose sensores de tipo RGB. Las altitudes de los vuelos fueron variables entre los $20 \mathrm{~m}$ y los $80 \mathrm{~m}$., todos ellos realizados a velocidad constante de $4 \mathrm{~m} / \mathrm{s}$ y toma de imágenes equidistante. Además, se han tenido en cuenta parámetros de la cámara, lente, y distribución de los puntos de apoyo.

En la planificación se consideró un solape longitudinal del 90\% y el transversal del 70\% entre cada fotograma. Para la realización del modelo, se seleccionaron un total de 788 imágenes de las obtenidas, descartando todas aquellas no útiles. Con ello, la precisión media obtenida fue de $\pm 2-3 \mathrm{~mm}$, siendo el parámetro Ground Sampling Distance (GSD) de 1,2 mm (Figura 10). 


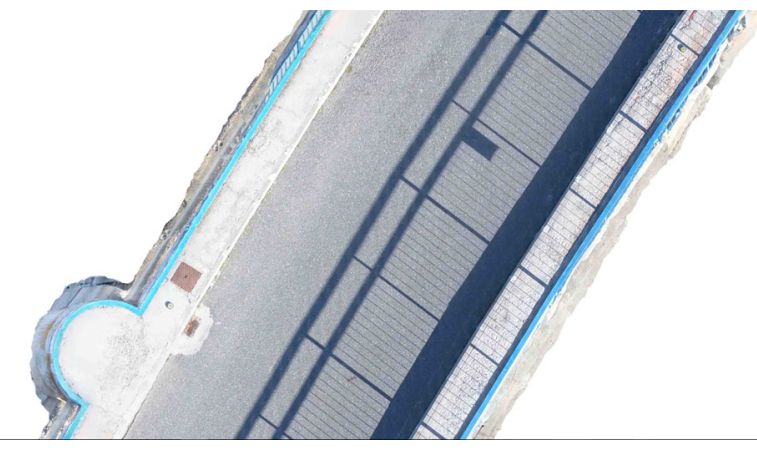

Figura 10: Detalle sobre Ortofotografía en planta con 1,2 mm/pixel de la Campaña 2 (feb-2018).

Las campañas fotogramétricas se realizaron en dos periodos diferentes del año, determinando el movimiento de la presa en ese periodo de tiempo y poder compararlo con los datos de la auscultación clásica de la presa. Las fechas en las que se realizaron fueron:

1. Campaña 1: Toma de datos y vuelo con dron entre el 24 y el 25 de octubre de 2017.

2. Campaña 2: Toma de datos y vuelo con dron entre el 18 y el 26 de febrero de 2018.

3. Campaña 3: Toma de datos y vuelo con dron el 19 de octubre del 2018.

Los GCPs utilizados en los modelos en planta fueron de dos tipologías, tal y como se puede apreciar en la Figura 11. La primera de ellas, consiste en placas metálicas con adhesivo amarillo y negro, estando el centro definido con una marca de $1 \mathrm{~mm}^{2}$. Se distribuyeron sobre ambas aceras de la presa con una equidistancia de $30 \mathrm{~m}$. La segunda ti-

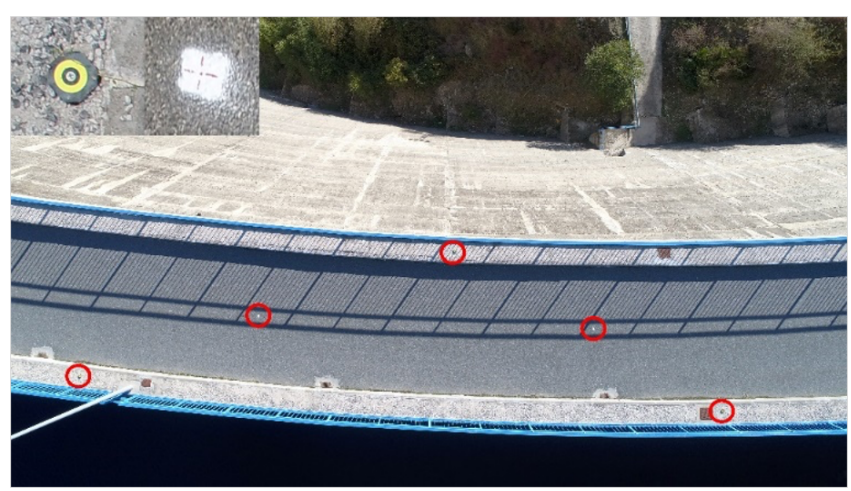

Figura 11. Tipología y distribución de GCPs. GCPs tipo placa distribuidos en las aceras y tipo marcas pintadas sobre el eje de la presa. pología, consistió en marcas pintadas sobre el eje de la presa, en las que también se marcó el centro de $1 \mathrm{~mm} \times 1 \mathrm{~mm}$. La distribución de los GCPs fue de forma alterna, con el objetivo de poder realizar diferentes combinaciones en el procesado. En el caso de los modelos realizados en alzado, se utilizaron como GCPs las propias dianas de auscultación de la presa.

Un buen resultado fotogramétrico, tiene su fundamento en una correcta toma de datos de campo, así como el conocimiento de los parámetros que intervienen en el procesado durante las campañas.

Así, en función de la campaña y el tipo de modelo, se recogen en la tabla 3 las características técnicas y meteorológicas con la siguiente leyenda : (1) GCPs tipo placa; (2) Dron Phantom 4 Pro con objetivo FOV84 $8.8 \mathrm{~mm} / 24 \mathrm{~mm}$ y sensor 1"CMOS; (3) Dron Matrice 210 RTK con cámara Zenmuse X4s, objetivo F/2.8 - 11, 8.8mm y sensor 1" CMOS; (4) Resolución fotográfica 5472x3078 pixeles; (5) Orientación cenital. (6) Orientación normal $\left(90^{\circ}\right)$.

\subsection{Procesado y resultados}

Las tareas en la fase de procesado fueron las siguientes:

1. Modelo general: Realización de un modelo general de la presa en 3D mediante un vuelo a alta cota (Figura 1).

2. Modelo coronación en planta: Determinación del modelo en planta de la coronación de la presa mediante vuelo a baja cota (Figura 10).

3. Modelo coronación en alzado: Definición del modelo del paramento aguas abajo desde la coronación a cota $-3 \mathrm{~m}$ (Figura 12).

4. Modelo 3D integrando alzado y planta: Modelo completo integrando planta y hasta cota -3 del paramento de la presa de aguas abajo.

Una vez procesados los modelos en ambas campañas, y obtenidas las ortofotografías, modelos $3 \mathrm{D}$ y nubes de puntos, se llevó a cabo tanto la comparativa entre ambas como el análisis de los resultados obtenidos. Es destacable la cantidad de puntos obtenida en los modelos con más de 100 millones de puntos cada uno.

Con objeto de verificar las diferencias entre mediciones topográficas y fotogramétricas, se llevó a cabo un control de calidad (Figura 13) basado en la comparativa de las coordenadas obtenidas para los clavos de nivelación existentes

Tabla 3. Programas de tratamiento de nubes de puntos y modelos 3D.

\begin{tabular}{|c|c|c|c|}
\hline & Modelo General & Modelo en Planta & Modelo en Alzado \\
\hline Campaña 1 & $\begin{array}{c}\text { (1),(2),(4),(5) } \\
\text { No Imágenes: } 177 \\
\text { Velocidad viento: } 2-3 \mathrm{~m} / \mathrm{s} \\
\text { Condición luz: soleado }\end{array}$ & $\begin{array}{c}\text { (1),(2),(4),(5) } \\
\text { No Imágenes: } 345 \\
\text { Velocidad viento: } 3-4 \mathrm{~m} / \mathrm{s} \text {. } \\
\text { Condición luz: soleado }\end{array}$ & $\begin{array}{c}\text { (2),(3),(4),(6) } \\
\mathrm{N}^{\circ} \text { Imágenes:118 } \\
\text { Velocidad viento: } 3-4 \mathrm{~m} / \mathrm{s} \text {. } \\
\text { Condición luz: soleado }\end{array}$ \\
\hline Campaña 2 & No realizado & 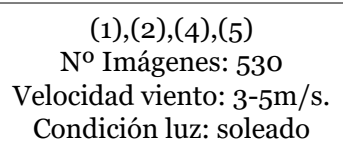 & $\begin{array}{c}\text { (2),(3),(4),(6) } \\
\text { No Imágenes: } 428 \\
\text { Velocidad viento: } 3-5 \mathrm{~m} / \mathrm{s} \text {. } \\
\text { Condición luz: soleado }\end{array}$ \\
\hline Campaña 3 & No realizado & $\begin{array}{c}\text { (1),(2),(4),(5) } \\
\text { No Imágenes: } 427 \\
\text { Velocidad viento: } 4 \mathrm{~m} / \mathrm{s} \text {. } \\
\text { Condición luz: nublado }\end{array}$ & $\begin{array}{c}\text { (2),(3),(4),(6) } \\
\text { No Imágenes: } 330 \\
\text { Velocidad viento: } 4 \mathrm{~m} / \mathrm{s} \text {. } \\
\text { Condición luz: nublado }\end{array}$ \\
\hline
\end{tabular}


en la presa por ambas metodologías. Evidentemente, estos datos topográficos no se introdujeron en el procesado fotogramétrico. Este control de calidad estimó unas diferencias entre mediciones en promedio de $1 \mathrm{~mm}$.

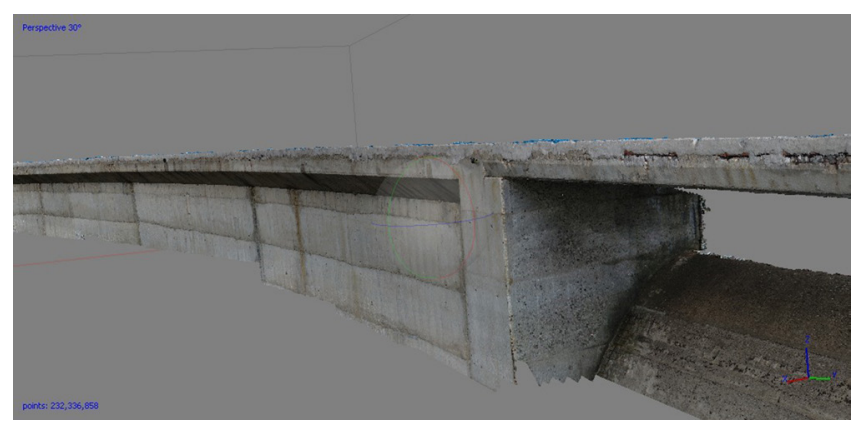

Figura 12. Nube de puntos del alzado del aliviadero (230 millones de puntos) generada en la Campaña 3 (oct-2018).

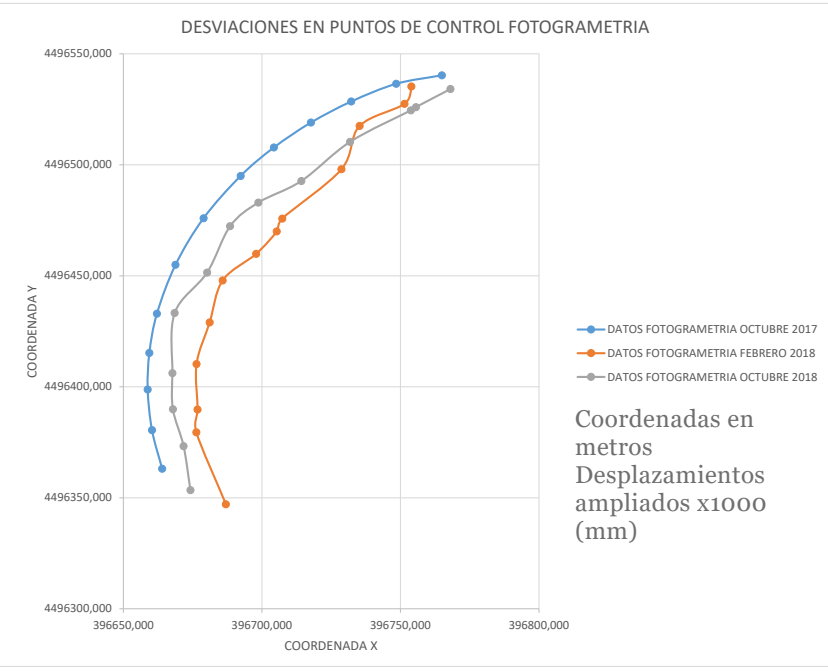

Figura 13. Control de calidad de la Campaña 2 (feb-2018) comparando coordenadas de los clavos de nivelación obtenidas mediante lecturas topográficas versus las obtenidas mediante fotogrametría.

Se presenta a continuación un primer resultado obtenido, en el que se puede ver el movimiento de la coronación de la presa arco-gravedad estudiada (Figuras 14 y 15). Se observa el movimiento tanto en $2 \mathrm{D}$, mediante la comparativa de las ortofotografías, como en $3 \mathrm{D}$, mediante la comparativa de las nubes de puntos.

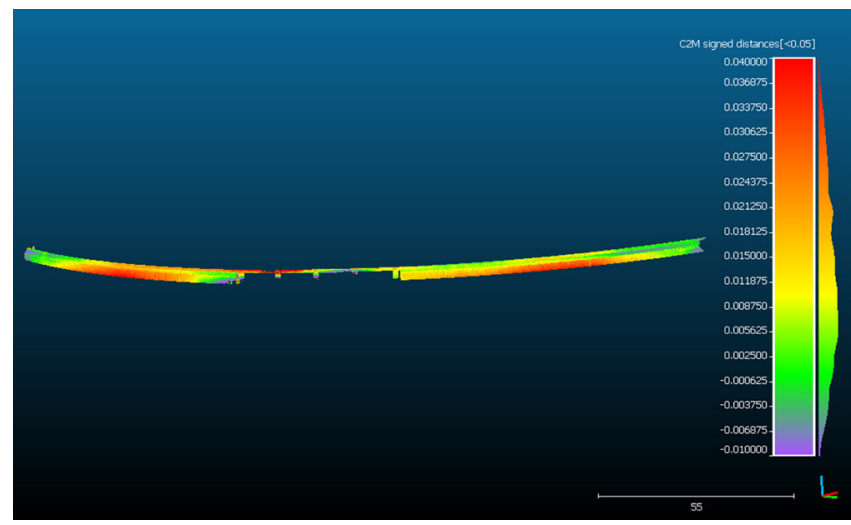

Figura 14. Deformación 3D de la coronación (m) entre las Campañas 1 (oct-2017) y 2 (feb-2018) y representación de la distribución de frecuencias (histograma).

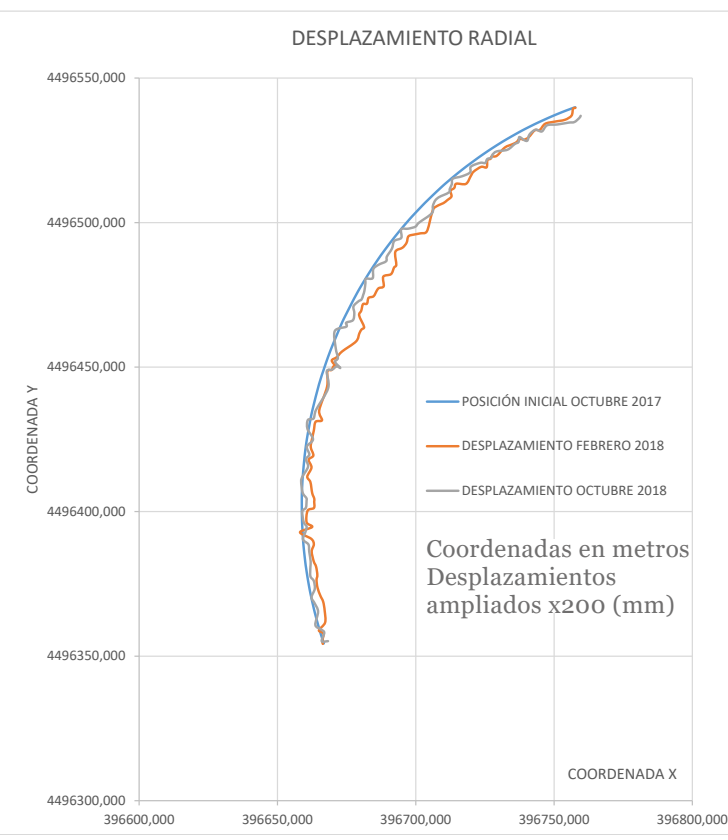

Figura 15. Deformación radial sobre planta de la presa. Coordenadas absolutas (m) y deformaciones ampliadas (x200). Campañas oct-2017, feb-2018, oct-2018.

\subsection{Aplicaciones al control deformacional geo- técnico}

La aplicación de la tecnología fotogramétrica con dron al caso de estudio de laderas es un aporte innovador de esta investigación, tal y como se ha visto previamente en el análisis documental realizado. En estudios geotécnicos, la precisión necesaria es de $\pm 3-4 \mathrm{~cm}$., siendo la accesibilidad el condicionante fundamental en el que basar un estudio de deformaciones, por lo que el uso de esta técnica es de gran utilidad.

El segundo caso de estudio corresponde a la auscultación del movimiento de una ladera (Figura 16). Para ello se realizó una Campaña específica para comparar con la primera. Los resultados obtenidos, en relación con el movimiento observado, se muestran en la Figura 17.

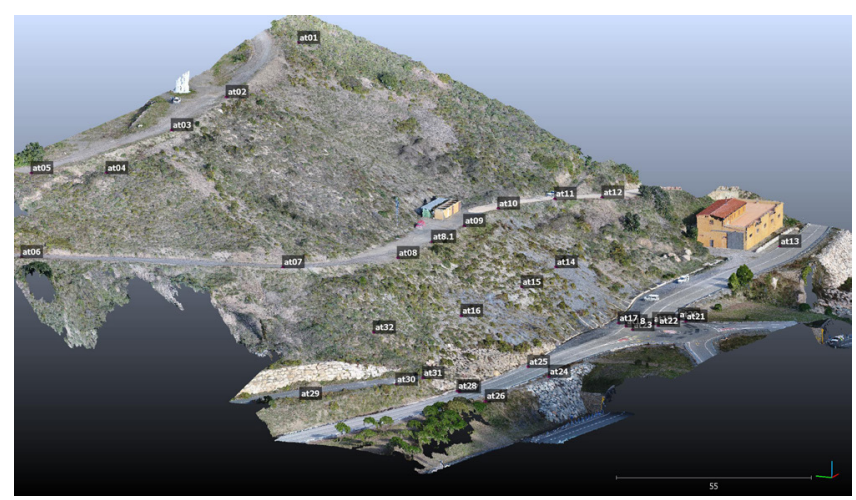

Figura 16. Nube de puntos densa georreferenciada de la ladera obtenida en la Campaña 1.

La precisión alcanzada al comparar la técnica fotogramétrica con la topografía clásica, es $2 \mathrm{~cm}$. Valor razonable para control de deformaciones asociados a laderas y estudios geotécnicos. 


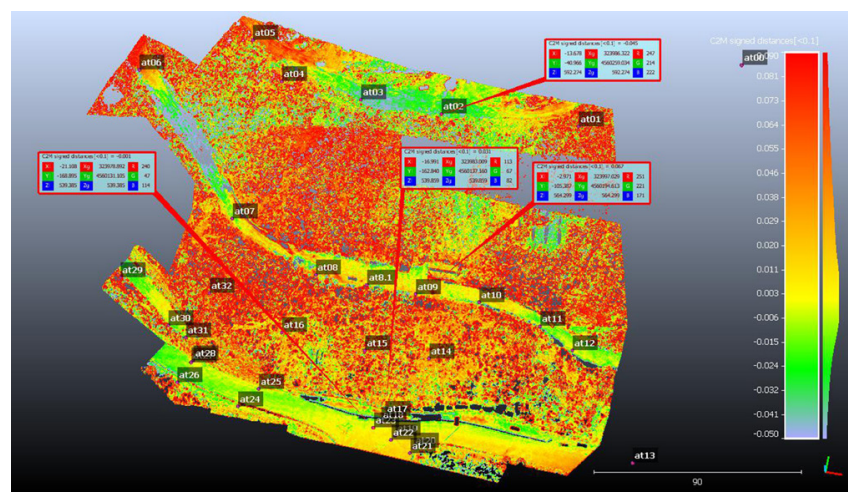

Figura 17. Mapa de deformaciones 3D obtenido para control de movimientos en ladera. Campaña 1 vs Campaña 3

\section{CONCLUSIONES}

La aplicación de fotogrametría mediante drones está provocando un salto no solo cuantitativo en la captura masiva de datos, sino también cualitativo. Las nubes de puntos densas y en color verdadero obtenidas de fachadas, elementos o estructuras, permiten, junto con la creación de una malla georreferenciada, la inspección global y continua del elemento así como el control de deformaciones y modelización del mismo. La automatización, simplicidad y cantidad de datos obtenidos mediante esta técnica, hacen que su aplicación se cada día mayor.
Según la revisión bibliográfica sistemática realizada, solamente dos artículos (6\%) presentan resultados de control de movimientos con tecnología dron, aplicándolo al monitoreo de un glaciar (23) y de una ladera con precisiones centimétricas $(9 \mathrm{~cm})(24)$. La presente investigación propone y aplica una metodología que permite controlar movimientos en estructuras y laderas con precisiones para el caso de presas arco-gravedad de $\pm 2-3 \mathrm{~mm}$.

Los factores determinantes constatados para obtener mayor resolución y precisión son: la disposición, forma y tipología de los puntos de control (GCPs), la distancia y ángulo de incidencia de la toma de imágenes, la resolución y estabilización del sensor, la incidencia solar y la tipología de geoposicionamiento utilizada por el dron. En este último aspecto, es destacable el uso de drones con sistema RTK, que permiten la reducción del número de GCPs.

La minimización de los costes y de los tiempos empleados en campo y oficina, hacen que esta técnica sea muy ventajosa frente a otras existentes en la actualidad. Asimismo, la galería de fotografías y videos obtenida permite la inspección visual en detalle.

Las líneas de investigación abiertas se centran en la integración de esta tecnología en los sistemas de auscultación oficiales de las infraestructuras, la combinación con otros sensores espectrales (i.e. térmico), la optimización en la distribución y tipología de GCPs y la integración en la modelización del complejo suelo-estructura.

\section{REFERENCIAS}

(1) Ministerio de Fomento, G. de E. (2018). Plan estrategico para el desarrollo del sector civil de drones en España 20182021. Madrid. España. 73 pp.

(2) Cuerno-Rejado, C., Garcia-Hernandez, L., Sanchez-Carmona, A., Carrio-Fernandez, A., Sanchez-Lopez, J., Campoy-Cervera, P. (2015). Evolution of the Unmanned Aerial Vehicles until present. DYNA Mayo 201590 (3): 281-288, doi: http:// dx.doi.org/10.6036/7781.

(3) Chabot, D. (2018). Trends in drone research and applications as the Journal of Unmanned Vehicle Systems turns five. Journal of Unmanned Vehicle Systems 6: vi-xv, doi https://doi.org/10.1139/juvs-2018-0005.

(4) Patterson, B. J. (2018). An Aerial View of the Future - Drones in Construction. Geospatialworld. https://www.geospatialworld.net/blogs/an-aerial-view-of-the-future-drones-in-construction/.

(5) Goldman Sachs (2018). The opportunity ahead. https://www.goldmansachs.com/insights/technology-driving-innovation/drones/.

(6) Nissen, E., Arrowsmith, J. R., \& Crosby, C. (2010). Introduction to Structure-from-Motion. Geological Society of America Annual Meeting Short Course, Denver, CO. https://kb.unavco.org.

(7) Furukawa, Y., \& Ponce, J. (2010). Accurate, dense, and robust multiview stereopsis. IEEE Transactions on Pattern Analysis and Machine Intelligence 32. Doi: https://doi.org/10.1109/TPAMI.2009.161.

(8) Souto-Vidal, M., Ortiz-Sanz, J., Gil-Docampo, M. (2015). Implementación del levantamiento eficiente de fachadas mediante fotogrametría digital automatizada y el uso de software gratuito. Informes de la Construcción, 67(539): e107, doi: http://dx.doi.org/10.3989/ic.14.098.

(9) Gonzalez-Rodrigo, B., Tendero-Caballero, R., Garcia-De Viedma, M., Pestana-Puerta, J., Carrio-Fernandez, A., Sanchez-Lopez, J., Suarez-Fernandez, R., Campoy-Cervera, P., Bonatti-Gonzalez, J., Rejas-Ayuga, J., Martinez-Marin, R., Marchamalo, M. (2016). Thermal UAV monitoring of facades: application for building rehabilitation. DYNA, 91(5). 571577. doi: http://dx.doi.org/10.6036/7899

(10) Hartley, R., \& Zisserman, A. (2000). Multiple View Geometry in Computer Vision. Cambridge University Press. Nueva York USA. ISBN: 978-0521623049. 624 pp.

(11) Ullman S. (1979). The Interpretation of Structure from Motion. Proceedings of the Royal Society of London. Series B, Biological Sciences 203(1153). doi: http://doi.org/10.1098/rspb.1979.0006

(12) Lowe, D. G. (1999). Object Recognition from Local Scale-Invariant Features. Proceedings of the Seventh IEEE International Conference on Computer Vision. 20-27 Sept. 1999. doi: 10.1109/ICCV.1999.790410

(13) Barberini, M., Rubboli, M. (2016) Il Rilievo fotogrammetrico con il Drone alla Diga di Ridracoli. GEOmedia 19 (6): 2283-5687. http://ojs.mediageo.it/index.php/GEOmedia/article/view/1280.

(14) Ridolfi, E., Buffi, G., Venturi, S. \& Manciola, P. (2017). Accuracy Analysis of a Dam Model from Drone Surveys. Sensors 17, 1777, doi:10.3390/s17081777

(15) Pagliari, D., Rossi L., Passoni, D., Pinto, L., De Michele, C. \& Avanzi, F. (2017) Measuring the volume of flushed sediments in a reservoir using multi-temporal images acquired with UAS, Geomatics, Natural Hazards and Risk, 8:1, 150166, doi: 10.1080/19475705.2016.1188423 
(16) Buffi, G., Manciola, P., De Lorenzis, L., Cavalagli, N., Comodini, F., Gambi, A., Gusella, V., Mezzi, M., Niemeier, W., Tamagnini, C. (2017). Calibration of finite element models of concrete arch-gravity dams using dynamical measures: The case of Ridracoli. Procedia Eng. 2017, 199, 110-115. https://doi.org/10.1016/j.proeng.2017.09.169

(17) Buffi, G., Manciola, P., Grassi, S., Gambi, A., Barberini, M. (2017). Survey of the Ridracoli Dam: UAV-Based Photogrammetry and Traditional Topographic Techniques in the inspection of Vertical Structures. Geomat. Nat. Hazards Risk 2017, 8: 1562-1579. https://doi.org/10.1080/19475705.2017.1362039

(18) Ridolfi, E.; Manciola, P. (2018). Water Level Measurements from Drones: A Pilot Case Study at a Dam Site. Water 2018, 10, 297,doi:10.3390/w10030297.

(19) Tillian M., Sulzer, W. (2015). Remote Sensing (UAV) for torrent inspection / survey in the alpine municipality of Weng im Gesäuse (Austria). 17th International Symposium "Landscape and Landscape Ecology”, 27-29 May 2015, Nitra, Slovakia. Editors: Martin Boltižiar \& Andrej Bača. Bratislava, Slovakia. ISBN 978-80-89325-27-6

(20) Kaufmann, V., Seier, G.. (2016). Long-Term Monitoring of Glacier Change at Gössnitzkees (Austria) Using Terrestrial Photogrammetry. The International Archives of the Photogrammetry, Remote Sensing and Spatial Information Sciences XLI-B8: 495-502, doi:10.5194/isprsarchives-XLI-B8-495-2016.

(21) Kaufmann, V., Seier, G., Sulzer, W., Wecht, M., Liu, Q., Lauk, G., and Maurer, M. (2018) Rock glacier monitoring using aerial photographs: conventional vs. UAV-based mapping - a comparative study. Int. Arch. Photogramm. Remote Sens. Spatial Inf. Sci., XLII-1: 239-246, doi: https://doi.org/10.5194/isprs-archives-XLII-1-239-2018, 2018

(22) Seier G., Stangl, S., Schöttl, S., Sulzer, W. \& Sass, O. (2017) UAV and TLS for monitoring a creek in an alpine environment, Styria, Austria. International Journal of Remote Sensing, 38:8-10, 2903-2920, doi: 10.1080/01431161.2016.1277045.

(23) Seier, G., Kellerer-Pirklbauer, A., Wecht, M., Hirschmann, S., Kaufmann, V., Lieb, G. K., and Sulzer, S. (2017). UAS-Based Change Detection of the Glacial and Proglacial Transition Zone at Pasterze Glacier, Austria. Remote Sensing 9: 549. doi:10.3390/rs9060549.

(24) Seier, G., Sulzer, W., Lindbichler, P., Gspurning, L., Hermann, S., Konrad, H.M., Irlinger, G. \& Adelwöhrer, R. (2018) Contribution of UAS to the monitoring at the Lärchberg-Galgenwald landslide (Austria), International Journal of Remote Sensing, 39:15-16, 5522-5549, doi: 10.1080/01431161.2018.1454627

(25) Gao, A., Wu, S., Wang, F., Wu, X., Xu, P., Yu, L., Zhu, S. (2019). A Newly Developed Unmanned Aerial Vehicle (UAV) Imagery Based Technology for Field Measurement of Water Level. Water 2019, 11, 124. doi:10.3390/w11010124

(26) Yu, J., Gan, Z., Zhong, L. \& Deng, L. (2018) Research and practice of UAV remote sensing in the monitoring and management of construction projects in riparian areas. ISPRS - International Archives of the Photogrammetry, Remote Sensing and Spatial Information Sciences, XLII-3, 2018: 2161-2165 doi: 10.5194/isprs-archives-XLII-3-2161-2018

(27) Li, Y., Gong, J. H., Zhu, J., Ye, L., Song, Y. Q., and Yue, Y. J.: Efficient dam break flood simulation methods for developing a preliminary evacuation plan after the Wenchuan Earthquake. Nat. Hazards Earth Syst. Sci., 12, 97-106, https:// doi.org/10.5194/nhess-12-97-2012, 2012.

(28) Yang, M., Huang, K. \& Tsai, H. (2016) Monitoring and Measurement of an Artificial Landslide Dam Using UAV Images and Image-Based Modeling. 2016 International Conference on Mathematical, Computational and Statistical Sciences and Engineering (MCSSE 2016)

(29) Zhang, S., Lv, P., Yang, X., Chen, X. \& Zhou, J. (2018). Spatiotemporal distribution and failure mechanism analyses of reservoir landslides in the Dagangshan reservoir, south-west China. Geomatics, Natural Hazards and Risk, 9:1, 791815, doi: 10.1080/19475705.2018.1480536

(30) US Society on Dams. (2016) US Committee on Monitoring of Dams and Their Foundations; USSD Committee on Levees. Monitoring Levees. 42 pp.

(31) Koparan, C., Koc, A. B., Privette, C. V \& Sawyer, C. B. (2018). In Situ Water Quality Measurements Using an Unmanned Aerial Vehicle ( UAV ) System. Water 2018, 10, 264. doi:10.3390/w10030264

(32) Khaloo A, Lattanzi D, Jachimowicz A and Devaney, C. (2018) Utilizing UAV and 3D Computer Vision for Visual Inspection of a Large Gravity Dam. Front. Built Environ. 4:31. doi: 10.3389/fbuil.2018.00031

(33) Matthew D. Larson, Simic Milas A., Vincent, R.K., \& Evans, J.E. (2018) Multi-depth suspended sediment estimation using high-resolution remote-sensing UAV in Maumee River, Ohio, International Journal of Remote Sensing, 39:15-16, 5472-5489, doi: 10.1080/01431161.2018.1465616

(34) Benson, J. et al. Microorganisms Collected from the Surface of Freshwater Lakes Using a Drone Water Sampling System (DOWSE). Water 2019, 11, 157. doi:10.3390/w11010157

(35) Jordá-Bordehore, L., Riquelme, A., Tomás, R. \& Cano, M. (2016). Análisis estructural y geomecánico en zonas inaccesibles de cavernas naturales mediante técnicas fotogramétricas: aplicación en la entrada de la cueva de Artá (Mallorca). El karst y el hombre las cuevas como Patrim. Mundial. 528 (2016).

(36) Tomás, R., Riquelme, A., Cano, M., Abellán, A, Jordá, L.. (2016). Structure from Motion (SfM): una técnica fotogramétrica de bajo coste para la caracterización y monitoreo de macizos rocosos. 10o Simp. Nac. Ing. Geotécnica, A Coruña, España 1, 209-216 (2016). ISBN 978-84-945284-2-2, págs. 209-216

(37) Villanueva, J. R. E., Martinez, L. I., \& Montiel, J. I. P. (2019). DEM Generation from Fixed-Wing UAV Imaging and LiDAR-Derived Ground Control Points for Flood Estimations. Sensors. 2019, 19, 3205. https://doi.org/10.3390/ S19143205 